\title{
Body Fat Accumulation is Associated with Early Arterial Changes in HIV- Infected Children
}

\author{
Marietta Charakida ${ }^{1 \# *}$, Agnieszka B Dzwonek ${ }^{3 \#}$, Vas Novelli ${ }^{2}$ and Nigel J Klein ${ }^{3}$ \\ ${ }^{1}$ Vascular Physiology Unit, Institute of Cardiovascular Sciences, UCL, London, UK \\ ${ }^{2}$ Clinical Infectious Diseases Unit, Great Ormond Street Children's Hospital NHS Trust, London, UK \\ ${ }^{3}$ Infectious Diseases and Microbiology Unit, Institute of Child Health, UCL University, London, UK \\ \#These authors have equally contributed to this study
}

\begin{abstract}
Background: Antiretroviral therapy (ART) has greatly improved clinical outcomes in human immunodeciency virus (HIV) infection in adults and children. However, emerging data link ART to clinical lipodystrophy and premature atherosclerosis. We examined the impact of body fat changes on blood pressure and endothelial function in HIV infected children with and without ART.

Methods: Central and peripheral body fat distribution was assessed in 68 HIV infected children. In all children blood pressure was measured and high resolution ultrasound was used to determine endothelium dependent dilatation (flow mediated dilatation-FMD) in the brachial artery.

Results: From the 68 HIV infected children, 46 were on ART. There was no evidence of clinical lipodystrophy with ART in this cohort. Total cholesterol was increased in children on ART $(p<0.001)$. FMD was significantly lower in those on ART compared to antiretroviral naive children $(p<0.01)$. Increased BMI and waist as well as peripheral body circumference measurements in the arm and thigh were associated with higher systolic blood pressure $(p<0.05$ and $p<0.01$ respectively). An inverse association was noted between peripheral body composition measurements (zcalf and zthigh) and FMD ( $p<0.05$ for both).

Conclusion: HIV-infected children on ART have deranged lipids and evidence of early arterial abnormalities. Increased body fat accumulation is associated with reduced endothelial function and elevated blood pressure in HIVinfected children. These findings suggest that healthy dietary intake and weight control would be advisable in HIV infected children for optimal vascular disease prevention.
\end{abstract}

Keywords: Body fat; Lipodystrophy; HIV; Endothelial function

\section{Introduction}

With the increased survival of HIV-infected patients, the development of cardiovascular disease is becoming a clinical concern [1]. Prospective and retrospective clinical trials have demonstrated that, particularly in the presence of cardiovascular risk factors (i.e. hypertension, diabetes, and smoking), HIV-infected adults have an increased incidence of cardiovascular events [2].

The cardiovascular risk in HIV- infected children remains low [3]. However, we and others, have demonstrated functional and structural changes consistent with accelerated atherosclerotic processes in children with HIV-infection [4-7]. The reasons for these changes are unclear and as in adults, appear to be related to disease severity, nature of antiretroviral therapy (ART) and immune activation.

Central obesity and lipid derangements are known to increase cardiovascular risk in the general population, but their impact in HIV-infected children is unknown [1]. Of particular concern are body fat changes (clinical lipodystrophy) described as peripheral lipoatrophy (subcutaneous adipose tissue wasting) and/or central lipohypertrophy (central fat accumulation) [8]. Depending on the methodology, up to a third of HIV infected children on ART show altered body fat distribution [8] . Ethnicity, duration of HIV infection, and ART all influence its frequency and severity [9]. These changes are aesthetically disturbing, and may herald a wider derangement of lipids and metabolic pathways as well as risk of cardiac disease [10]. In addition, body fat changes in children are of particular concern as data in adults suggest that the presence of clinical lipodystrophy is associated with a significantly higher risk of coronary heart disease at 10 years as assessed by the Framingham risk score, than patients without clinical lipodystrophy [11]. However the relative importance of HIV disease, ART and associated body fat changes remains unclear and is particularly challenging to unravel in adults who have multiple additional cardiovascular risk factors. We therefore assessed the impact of body fat changes on markers of early arterial disease i.e. endothelial function and blood pressure in children with HIV infection with and without antiretroviral therapy.

\section{Methods}

\section{Study population}

We studied HIV-infected children attending Great Ormond Street Hospital (GOSH) NHS Trust (London, UK). Children older than 6 years of age who could cooperate and stay still for vascular assessment were eligible to participate in this study. Children with current opportunistic infections or cardiovascular risk factors, including

${ }^{*}$ Corresponding author: Marietta Charakida, Academic Clinical Lecturer in Paediatric Cardiology, Institute of Cardiovascular Sciences, UCL170 Tottenham Court Road, London W1T 7HA, UK, Tel: 0044 (0)2076799404; E-mail: charakidadoc@gmail.com

Received July 30, 2014; Accepted September 21, 2014; Published October 03 , 2014

Citation: Charakida M, Dzwonek AB, Novelli V, Klein NJ (2014) Body Fat Accumulation is Associated with Early Arterial Changes in HIV-Infected Children. J AIDS Clin Res 5: 356. doi:10.4172/2155-6113.1000356

Copyright: (C) $2014 \mathrm{Nel} \mathrm{AM}$, et al. This is an open-access article distributed unde the terms of the Creative Commons Attribution License, which permits unrestricted use, distribution, and reproduction in any medium, provided the original author and source are credited. 
hypertension, diabetes, and renal failure, were not eligible for this study. None had a current or recent infectious illness, nor were they receiving any medication or vitamin supplementation. Details of past clinical and ART history were extracted from the UK Collaborative HIV Pediatric Study (CHIPS) database [12]. Children were started on ART, for low or declining CD4\% counts, as defined by PENTA guidelines and/or clinical deterioration [13]. The nature of the ART regime was not based on measures of disease severity.

Ethnicity data were obtained from the National Study of HIV in Pregnancy and Childhood (NSHPC). The study protocol was approved by the Institute of Child Health Ethics Committee. All parents or caregivers gave written informed consent and assent was taken from the children when this was appropriate.

\section{Cardiovascular risk assessment}

Clinical assessment: Systolic and diastolic blood pressures were measured as the average of three seated readings with an automated oscillometric device (Omron) in the left arm. A paediatric cuff was used when the arm circumference was $<25 \mathrm{~cm}$ and a regular adult cuff when the arm circumference was $>25 \mathrm{~cm}$. Heart rate was measured as the average of the last two readings as these have been recorded by the same (Omron) device.

Vascular measurements: Endothelium-dependent vascular response was assessed in the right brachial artery by high-resolution ultrasound imaging using an Acuson 5 to $10 \mathrm{MHz}$ linear probe as previously described [14]. Doppler-derived flow measurements (using a pulse wave Doppler signal at a $70^{\circ} \mathrm{C}$ angle) were also obtained continuously. Brachial artery diameter was measured offline by an automatic edge detection system (Brachial Tools) and expressed as a percentage change from the baseline diameter. Endotheliumindependent response to a $25 \mu \mathrm{g}$ dose of sublingual glyceryl trinitrate (GTN) was also calculated as a percentage change from the baseline diameter.

Biochemical markers: Non-fasting blood was taken in heparinized tubes and $1 \mathrm{~mL}$ of plasma was stored at $-80^{\circ} \mathrm{C}$. Lipid levels (total cholesterol, HDL, non-HDL cholesterol, triglycerides and lipoprotein alpha) and high sensitivity CRP (hsCRP) were measured. Disease activity was also assessed by viral load and CDC stage.

Anthropometric measurements: Weight was measured to the nearest $0.1 \mathrm{~kg}$ using Tanita scales (Wardworth Ltd, Bolton, United Kingdom). Height was measured to the nearest $0.1 \mathrm{~cm}$ using a Harpenden stadiometer (Holtain Ltd, Crymych Pembrokeshire, United Kingdom) and body mass index (BMI $\mathrm{kg} / \mathrm{m}^{2}$ ) was calculated. Waist circumference was measured using a flexible tape (Chasmors Ltd, London, UK), to the nearest $1 \mathrm{~mm}$, at the midpoint between the lower ribs and the pelvic bone, and the ratio of waist circumference to height was calculated. This has been validated as a measure of central adiposity in children. The values were converted to age- and sex-adjusted z-scores using the UK reference curves. Triceps, biceps, subscapular and suprailiac skinfold measurements were taken using a Harpenden Skinfold Caliper (Holtain Ltd,Crymych, UK). Mid-upper arm, thigh and calf circumferences were measured using a narrow $1-\mathrm{m}$ tape (Chasmors Ltd, London, UK). All skinfold and circumference measurements were performed by the same trained researcher (AD) in triplicate. Skinfold and circumference measurements were converted to age- and sex- adjusted z-scores as previously described [12].

We assessed central fat accumulation by using the following measurements: waist circumference, waist and hip ratio and subscapular skinfold thickness [15]. Peripheral fat distribution (fat accumulation or wasting) was assessed by measuring upper and lower limb circumferences (mid-upper arm, calf and thigh) and also by assessing skinfold thickness (triceps, biceps, and suprailiac) [16].

\section{Statistical analysis}

In descriptive analyses, parametric summary statistics and significance tests were used when the data were approximately normally distributed; otherwise non-parametric methods were used. In normally distributed data values are expressed as mean (SD) unless otherwise stated.

Multivariable linear regression analysis was used to examine relationships between cardiovascular measurements and body fat composition following adjustment for potential confounders. Confounders were considered age, sex, viral load, antiretroviral treatment and biochemical measures, which were univariately associated with the dependent variable. Variables that were not normally distributed were $\log _{10}$ transformed to reduce the influence of outlying observations.

\section{Results}

Sixty-eight children of Sub-Saharan African origin agreed to participate in the study and underwent detailed vascular and anthropometric characterization. Fourty six were exposed to antiretroviral therapy. Triple ART therapy was used containing 2 nucleoside reverse transcriptase inhibitors (NRTIs) and a nonnucleoside reverse transcriptase inhibitor (NNRTI) or protease inhibitor $(\mathrm{N}=20)$ in their combination. Twenty-two children were antiretroviral naive. The median ART exposure was 5.04 years. HIVinfected children on ART had more advanced CDC stage and reduced viral load compared to untreated children (Table 1).

\section{Vascular phenotype in HIV infected children (Table 1)}

Systolic and diastolic blood pressure and heart rate were similar in HIV infected treated and untreated children. Baseline vessel diameter, baseline brachial blood flow and reactive hyperemia were similar in the different treatment group. FMD was significantly lower in those on ART that included a PI as part of the regimen 6.2 (4.3)\% compared to $8.3(3.4) \%$ without PI and 10.1 (4.2)\% in untreated children. The endothelium independent response to GTN was similar in the different treatment groups. There was no relation between blood pressure, viral load or lipids and FMD in adjusted or unadjusted models.

\section{Vascular phenotype and body fat changes in HIV-infected children}

There was a trend towards decreased peripheral body fat measurements in HIV-infected children who were on ART. Increased BMIz and waist (beta: 0.3 for both, $\mathrm{p}<0.05$ ) as well as peripheral body circumference measurements in the arm and thigh (beta: 0.3, $\mathrm{p}<0.05$ and beta: $0.4, \mathrm{p}<0.01$ respectively) were associated with higher systolic blood pressure (Figure 1).

An inverse association was noted between body composition measurements and FMD. Peripheral body composition measurements (zcalf and zthigh) had an inverse relationship with FMD (beta $=-0.3$ and beta: $-0.2, \mathrm{p}<0.05$ for both). These associations remained following adjustment for age, sex, baseline diameter, viral load and ART (Figure 2). 
Citation: Charakida M, Dzwonek AB, Novelli V, Klein NJ (2014) Body Fat Accumulation is Associated with Early Arterial Changes in HIV-Infected Children. J AIDS Clin Res 5: 356. doi:10.4172/2155-6113.1000356

Page 3 of 5

\begin{tabular}{|c|c|c|c|}
\hline & $\begin{array}{l}\text { Treated } \\
(\mathrm{n}=46)\end{array}$ & $\begin{array}{l}\text { Untreated } \\
(n=22)\end{array}$ & p value \\
\hline \multicolumn{4}{|l|}{ Demographic data } \\
\hline Age (years) & $11.4(2.9)$ & $10.7(2.9)$ & 0.3 \\
\hline Male \% (N)†† & $52(24)$ & $59(13)$ & 0.1 \\
\hline \multicolumn{4}{|l|}{ Clinical parameters } \\
\hline Weight & $36.2(11.9)$ & $37.9(14.6)$ & 0.6 \\
\hline BMI & $17.8(2.7)$ & $18.8(3.1)$ & 0.2 \\
\hline Systolic blood pressure (mmHg) & $102.4(9.2)$ & $107.0(10.3)$ & 0.08 \\
\hline Diastolic blood pressure $(\mathrm{mmHg})$ & $60.5(10.2)$ & $62.5(5.9)$ & 0.3 \\
\hline Heart rate $(\mathrm{bpm})$ & $80(11)$ & $84(16)$ & 0.2 \\
\hline Glucose (mmol/L) & $4.4(0.7)$ & $4.5(0.8)$ & 0.7 \\
\hline $\begin{array}{l}\text { High sensitivity CRP (mg/L); median } \\
\text { [IQR] }\end{array}$ & $1.1[0.2,2.4]$ & $0.4[1.4,2.4]$ & 0.3 \\
\hline $\mathrm{CDC}$ stage $(\mathrm{A} / \mathrm{C}) \dagger \dagger$ & $14 / 17$ & $13 / 0$ & 0.001 \\
\hline Viral load median[IQR] & $50[50,2175]$ & $\begin{array}{l}17400[11050 \\
36600]\end{array}$ & 0.0001 \\
\hline \multicolumn{4}{|l|}{ Lipid/ Blood results } \\
\hline Total cholesterol (mmol/L) & $4.3(0.9)$ & $3.5(0.8)$ & 0.002 \\
\hline HDL cholesterol (mmol/L) & $1.4(0.5)$ & $0.9(0.3)$ & 0.0001 \\
\hline Lp (a) (mg/L); median [IQR] & $46.1[30.6,77.7]$ & $34.7[22.6,67.3]$ & 0.5 \\
\hline Triglycerides (mmol/L); median [IQR] & $1.0[0.8,1.3]$ & $0.9[0.6,1.4]$ & 0.7 \\
\hline \multicolumn{4}{|c|}{ Anthropometric measurements z-scores (median [IQR] } \\
\hline Waist circumference & $0.2[-0.3,0.6]$ & $0.6[0.3,0.8]$ & 0.2 \\
\hline Arm circumference & $-0.7[-1.3,0.3]$ & $-0.2[-1.1,0.6]$ & 0.3 \\
\hline Thigh circumference & $-1.1[-2.0,0.1]$ & $-0.6[-1.2,-0.1]$ & 0.1 \\
\hline Calf circumference & $-0.8[-2.2,0.1]$ & $-0.9[-1.8,0.2]$ & 0.4 \\
\hline Biceps skin-fold & $1.1[0.2,1.9]$ & $1.9[0.8,3.8]$ & 0.05 \\
\hline Triceps skin-fold & $0.1[-0.5,1.1]$ & $0.8[-0.1,1.8]$ & 0.2 \\
\hline Subscapular skin-fold & $1.8[0.2,2.9]$ & $1.7[0.3,4.1]$ & 0.9 \\
\hline Suprailiac skin-fold & $-0.5[-1.1,-0.1]$ & $-0.6[-.9,0.4]$ & 0.3 \\
\hline \multicolumn{4}{|l|}{ Vascular measurements } \\
\hline Flow mediated dilation (\%) & $7.3(3.9)$ & $10.1(4.2)$ & 0.01 \\
\hline Baseline diameter (mm) & $2.7(0.5)$ & $2.8(0.5)$ & 0.7 \\
\hline Glyceryltrinitrate mediated dilation (\%) & $13.8(5.6)$ & $15.8(6.3)$ & 0.2 \\
\hline
\end{tabular}

The use of ART has changed the course of HIV disease from a terminal pathology to a chronic illness. However, new pathologies have appeared as patients continue to live with the virus and among them increased incidence of cardiovascular events with ART have been of particular interest [17]. However, despite numerous clinical trials in adults it remains
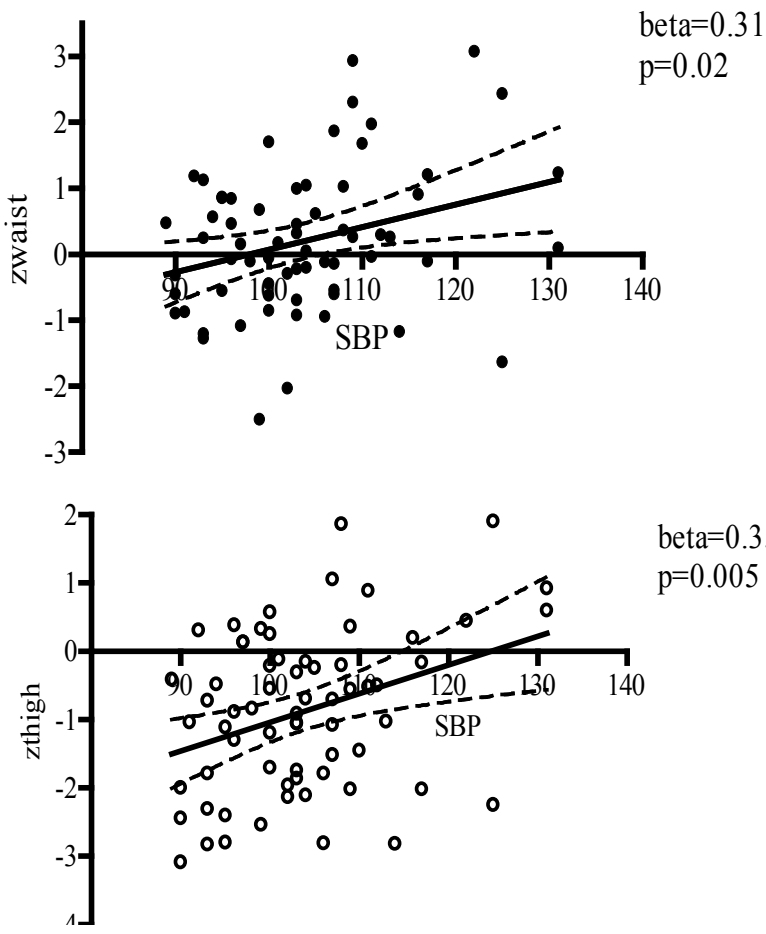

beta $=0.35$

$\mathrm{p}=0.005$

Figure 1: The impact of body fat distribution on systolic blood pressure in HIV infected children.

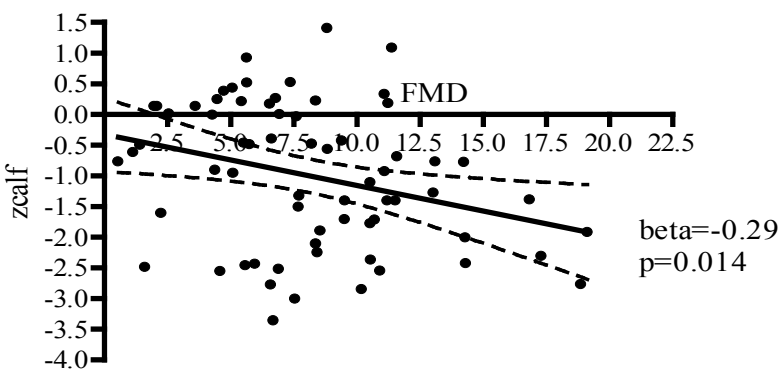

None of the lipid measurements were associated with body composition measurements irrespective of ongoing ART. Total cholesterol was increased in children on ART compared to untreated children (Table 1). In contrast HDL cholesterol was lower in the untreated HIV-infected children compared to ART treated (Table 1).

\section{Discussion}

In this study we demonstrated that HIV infected children on ART have evidence of early arterial changes with decreased endothelial function and increased systolic blood pressure and deranged lipid profile. We also demonstrated for the first time that these arterial changes are associated with increased fat accumulation rather than clinical lipodystrophy. These findings indicate that the general advice for healthy dietary intake and weight control should be advocated in HIV infected children to minimise later cardiovascular risk.

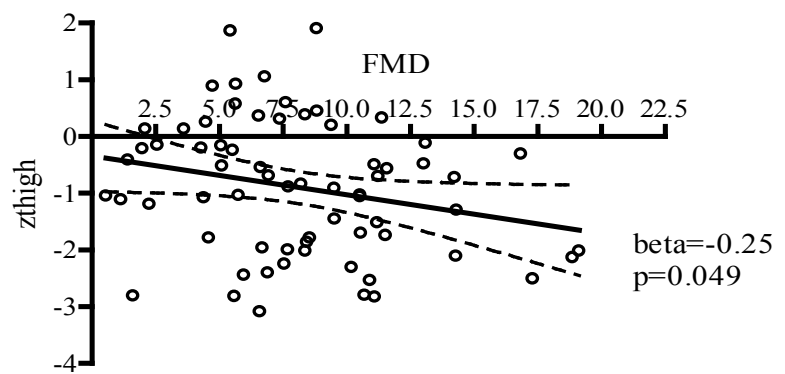

Figure 2: The impact of body fat distribution on endothelial function in HIVinfected children.

Anthropometric measurements had the same pattern of association with flow mediated dilatation with zcalf and zthigh being the strongest predictors in univariate and multivariate analysis. 
Citation: Charakida M, Dzwonek AB, Novelli V, Klein NJ (2014) Body Fat Accumulation is Associated with Early Arterial Changes in HIV-Infected Children. J AIDS Clin Res 5: 356. doi:10.4172/2155-6113.1000356

Page 4 of 5

difficult to disentangle the individual contribution of the virus itself, that of antiretroviral treatment and its associated metabolic abnormalities on individual's composite cardiovascular risk assessment [17].

In the general population, cardiovascular risk assessment is traditionally performed in adults by measuring a range of metabolic parameters in the circulation, including lipids and glucose as well as assessing clinical and environmental parameters such as hypertension, smoking, obesity and physical inactivity [18]. However, the low prevalence of these abnormalities in the young renders cardiovascular risk stratification challenging. In this study we decided to measure blood pressure, which although limited by greater variability, has consistently been shown to reflect cardiovascular risk and to predict later cardiovascular outcome $[19,20]$. We also assessed endothelial function by measuring FMD in the brachial artery to detect early functional arterial changes [14]. Reduced FMD denotes reduced nitric oxide bioavailability and numerous reports have shown that the technique is sensitive to detect early arterial changes in children at increased cardiovascular risk which can be reversed with appropriate treatment [21-23]. Consistent with our previous observations [5], in this study we found impaired endothelial function in HIV infected children who had more severe disease and were on ART. We were also able to demonstrate evidence of arterial changes with increasing central and peripheral body composition measurements.

Body fat abnormalities occur in the course of HIV disease in the presence and absence of ART [24]. We assessed body fat distribution by using a number of anthropometric measurements including measurement of skinfold thickness. This technique is easy to perform but is subject to operator variability. To minimise this potential error, the same trained operator performed all measurements in this study. More objective measures of subcutaneous fat stores and visceral fat include DEXA scanning, computed tomography and magnetic resonance imaging. However, these techniques are expensive and unlikely to be widely available as screening tools, particularly in the setting of HIV prevalence. In this study we were not able to detect significant body fat composition changes with ART in HIV-infected children in contrast to previous reports [13]. It is possible that this was due to the sample size in this study. However, we were able to detect associations between body composition measurements and arterial phenotype in all HIV patients, irrespective of ART. However these findings should be interpreted with caution as this study was cross sectional and therefore causal inferences cannot be identified.

The mechanisms by which increased body fat measurements might affect blood pressure and endothelial function in HIV-infected children remains unclear. We can only hypothesize that as in the general population, increased central and peripheral body fat might promote insulin resistance, sodium retention, increased sympathetic nervous system activity, immune activation and activation of renin-angiotensinaldosterone system [25]. These changes have consistently been shown to have a dynamic role in the development of hypertensive state and to promote inflammatory and haemostatic changes, which impair endothelial integrity and disturb its function $[26,27]$.

In summary, the data from this study suggests that central and peripheral body fat composition is associated with early arterial changes in HIV infected children. As cardiovascular prevention remains one of the main late concerns for this population our findings indicate that efficient cardiovascular prevention should not merely concentrate on treating the metabolic abnormalities associated with ART in HIV infected children, but on providing advice for a healthy lifestyle with attention on weight control.

\section{References}

1. Fedele F, Bruno N, Mancone M (2011) Cardiovascular risk factors and HIV disease. AIDS Rev 13: 119-129.

2. Ho JE, Hsue PY (2009) Cardiovascular manifestations of HIV infection. Heart 95: 1193-1202.

3. Werner ML, Pone MV, Fonseca VM, Chaves CR (2010) Lipodystrophy syndrome and cardiovascular risk factors in children and adolescents infected with HIVIAIDS receiving highly active antiretroviral therapy. J Pediatr (Rio J) 86: $27-32$

4. Charakida M, Loukogeorgakis SP, Okorie MI, Masi S, Halcox JP, et al. (2009) Increased arterial stiffness in HIV-infected children: risk factors and antiretroviral therapy. Antivir Ther 14: 1075-1079.

5. Charakida M, Donald AE, Green H, Storry C, Clapson M, et al. (2005) Early structural and functionalchanges of the vasculature in HIV-infected children impact of disease and antiretroviral therapy. Circulation 112: 103-109.

6. Bonnet D, Aggoun Y, Szezepanski I, Bellal N, Blanche S (2004) Arterial stiffness and endothelial dysfunction in HIV-infected children. AIDS 18: 1037-1041.

7. Sainz T, varez-Fuente M, Navarro ML, Diaz L, Rojo P, et al. (2014) Subclinical atherosclerosis and markers of immune activation in HIV-infected children and adolescents: the CaroVIH Study. J Acquir Immune Defic Syndr 65:42-49.

8. Magkos F, Mantzoros CS (2011) Body fat redistribution and metabolic abnormalities in HIV-infected patients on highly active antiretroviral therapy: novel insights into pathophysiology and emerging opportunities for treatment. Metabolism 60: 749-753.

9. Veloso S, Peraire J, Vilades C, Lopez-Dupla M, Escote X, et al. (2010) Pharmacogenetics of the metabolic disturbances and atherosclerosis associated with antiretroviral therapy in HIV-infected patients. Curr Pharm Des 16:3379-3389.

10. Sainz T, Diaz L, Navarro ML, Rojo P, Blazquez D, et al. (2014) Cardiovascular biomarkers in vertically HIV-infected children without metabolic abnormalities. Atherosclerosis 233:410-414

11. Freitas P, Carvalho D, Souto S, Santos AC, Xerinda S, et al. (2011) Impact of Lipodystrophy on the prevalence and components of metabolic syndrome in HIV-infected patients. BMC Infect Dis 11: 246

12. Gibb DM, Duong T, Tookey PA, Sharland M, Tudor-Williams G, et al. (2003) Decline in mortality, AIDS, and hospital admissions in perinatally HIV-1 infected children in the United Kingdom and Ireland. BMJ 327: 1019.

13. PENTA Steering Committee, Welch $S$, Sharland M, Lyall EG, Tudor-Williams $G$, et al. (2009) PENTA 2009 guidelines for the use of antiretroviral therapy in paediatric HIV-1 infection. HIV Med 10: 591-613.

14. Donald AE, Halcox JP, Charakida M, Storry C, Wallace SM, et al. (2008) Methodological approaches to optimize reproducibility and power in clinical studies of flow-mediated dilation. J Am Coll Cardiol 51: 1959-1964.

15. Dzwonek AB, Lawson MS, Cole TJ, Novelli V (2006) Body fat changes and lipodystrophy in HIV-infected children: impact of highly active antiretrovira therapy. J Acquir Immune Defic Syndr 43: 121-123.

16. Davidson LE, Wang J, Thornton JC, Kaleem Z, Silva-Palacios F, et al. (2011) Predicting fat percent by skinfolds in racial groups: Durnin and Womersley revisited. Med Sci Sports Exerc 43: 542-549.

17. Miller CJ, Baker JV, Bormann AM, Erlandson KM, Huppler Hullsiek K, et al. (2014) Adjudicated morbidity and mortality outcomes by age among individuals with HIV infection on suppressive antiretroviral therapy. PLoS One 9: e95061.

18. Tralhão A, Sousa PJ, Ferreira AM, Miranda M, Monge JC, et al. (2014) Cardiovascular risk profile of young adults: changes over time. Rev Port Cardio 33: $147-154$

19. Berenson GS, Srinivasan SR, Bao W, Newman WP 3rd, Tracy RE, et al. (1998) Association between multiple cardiovascular risk factors and atherosclerosis in children and young adults. The Bogalusa Heart Study. N Engl J Med 338 1650-1656.

20. Ruilope LM, Schmieder RE (2008) Left ventricular hypertrophy and clinical outcomes in hypertensive patients. Am J Hypertens 21: 500-508.

21. Celermajer DS, Sorensen KE, Bull C, Robinson J, Deanfield JE (1994) Endothelium-dependent dilation in the systemic arteries of asymptomatic subjects relates to coronary risk factors and their interaction. J Am Coll Cardio 24: $1468-1474$ 
Citation: Charakida M, Dzwonek AB, Novelli V, Klein NJ (2014) Body Fat Accumulation is Associated with Early Arterial Changes in HIV-Infected Children. J AIDS Clin Res 5: 356. doi:10.4172/2155-6113.1000356

22. Sorensen KE, Celermajer DS, Georgakopoulos D, Hatcher G, Betteridge DJ, et al. (1994) Impairment of endothelium-dependent dilation is an early event in children with familial hypercholesterolemia and is related to the lipoprotein (a) level. J Clin Invest 93: 50-55.

23. Mullen MJ, Clarkson P, Donald AE, Thomson H, Thorne SA, et al. (1998) Effect of enalapril on endothelial function in young insulin-dependent diabetic patients: a randomized, double-blind study. J Am Coll Cardiol 31: 1330-1335.

24. Arpadi SM, Bethel J, Horlick M, Sarr M, Bamji M, et al. (2009) Longitudinal changes in regional fat content in HIV-infected children and adolescents. AIDS 23: 1501-1509.
25. Singh M, Mensah GA, Bakris G (2010) Pathogenesis and clinical physiology of hypertension. Cardiol Clin 28: 545-559.

26. Flynn JT, Falkner BE (2011) Obesity hypertension in adolescents: epidemiology evaluation, and management. J Clin Hypertens (Greenwich) 13: 323-331.

27. Tziomalos K, Athyros VG, Karagiannis A, Mikhailidis DP (2010) Endothelial dysfunction in metabolic syndrome: prevalence, pathogenesis and management. Nutr Metab Cardiovasc Dis 20: 140-146. 